Please do not remove this page

RMIT

UNIVERSITY

\title{
Sensitivity improved photonic instantaneous frequency measurement receiver
}

Sarkhosh, Niusha; Emami, Hossein; Ghorbani, Kamran; Bui, Lam; Mitchell, Arnan

https://researchrepository.rmit.edu.au/esploro/outputs/9921863964601341/filesAndLinks?institution=61RMIT_INST\&index=null

Sarkhosh, N., Emami, H., Ghorbani, K., Bui, L., \& Mitchell, A. (2008). Sensitivity improved photonic instantaneous frequency measurement receiver. Proceedings from 2008 Asia-Pacific Microwave Conference, 1-4. https://doi.org/10.1109/APMC.2008.4958676

Published Version: https://doi.org/10.1109/APMC.2008.4958676

Repository homepage: https://researchrepository.rmit.edu.au

(c) 2008 IEEE. Personal use of this material is permitted. However, permission to reprint/republish this material for advertising or promotional purposes or for creating new collective works for resale or redistribution to servers or lists, or to reuse any copyrighted component of this work in other works must be obtained from the IEEE.

Downloaded On 2023/04/26 21:57:46 +1000 


\author{
Sensitivity Improved Photonic Instantaneous Frequency \\ Measurement Receiver \\ Niusha Sarkhosh, Hoseein Emami, Kamran Ghorbani, Lam Bui \\ and Arnan Mitchell \\ Department of Electrical and Computer Engineering, RMIT University, \\ Melbourne Australia \\ niusha.sarkhoh@rmit.edu.au
}

\title{
I. Introduction
}

FREQUENCY measurement is a critical component of modern electronic warfare systems. The Instantaneous Frequency Measurement (IFM) system has been widely used in electronic warfare for many years as these receivers detect the frequency of threat signals and provide an early indication of threat classification and also suggest frequency ranges in which to focus processing resources.

Traditional IFM receivers have been implemented simply using interferometers formed from hybrid couplers and delay lines [1-4].

On the other hand, microwave-photonics has been suggested as a means of increasing the bandwidth of signal processing systems [5-7]. Therefore it has been investigated for photonic IFM implementation [5]. The general concept is to use broad-band, low noise optical modulators to up-convert the RF signal to the optical domain, wide-band process in the optical domain, transmit it via optical fiber and then down-convert it to the RF.

Most receivers are implemented in a channelized configuration in order to increase the sorting capability of the system particularly in a dense environment. In order to realize multichannel IFM receivers in the optical domain, the receiver would require a separate photo detector for each IFM. The cost of multiple broadband photo detectors would be prohibitive expensive [5]. Recently we have explored a frequency measurement system where frequency detection is achieved via D.C measurement [8]. However the sensitivity of the receiver was poor due to high noise floor. In this paper we present a lock-in approach to reduce the noise hence better sensitivity.

\section{IFM Concept}

Fig. 1 (inset) shows a block diagram of a possible IFM system. A single RF tone is divided into two equal portions. One portion is delayed relative to the other by time $\tau$.The two RF signals are then multiplied together and the result is low-pass filtered.

Mathematically, the output of Fig. 1 can be written:

$$
\begin{aligned}
& V_{\text {out }}(t)=\frac{1}{4} V_{o}^{2} \cos \Omega t \cos \Omega(t-\tau) \\
& V_{\text {out }}(t)=\frac{1}{8} V_{o}^{2}[\cos (2 \Omega t-\Omega \tau)+\cos \Omega \tau]
\end{aligned}
$$

Where $V_{\mathrm{o}}$ and $\Omega$ are the amplitude and angular frequency of the RF signal and $\tau$ is the relative delay between the two paths. As it can be seen $V_{\text {out }}(\mathrm{t})$, has a frequency dependent DC portion of $\frac{1}{8} V_{0}^{2} \cos \Omega \tau$. 
Having established a relation between the frequency and output DC component, we are now able to design an instantaneous frequency measurement system which is able to predict the frequency of an RF signal using only a DC component.

\section{Photonic IFM}

Fig. 1 shows the schematic diagram of the proposed photonic IFM system. An RF signal generator produces a single RF tone which is divided equally into two portions using a Wilkinson power divider feeding two arms of the IFM system.

The RF signal is input to a Mach-Zehnder Modulator (MZM1) biased at quadrature $\left(V_{q}\right)$. MZM1 modulates an optical carrier with wavelength of $\lambda_{1}$ produced by a laser diode. The modulated carrier then traverses a fiber patch cord and experiences an optical delay. The second portion of the RF signal is input to a second modulator (MZM2) (biased at $V_{q}$ ) modulating the optical carrier a second time. The twice-modulated signal is detected by a photo-detector. The output of the photo-detector is then low-pass filtered and measured by a digital voltmeter.

The DC voltage measured by the digital voltmeter can be describes as:

$$
V_{D C}(\Omega) \approx \frac{1}{4} G Z_{P D} P_{o}\left(1+\frac{\pi^{2} Z_{i n} P_{R F}}{2 V_{\pi}^{2}}\right)+\frac{\pi^{2}}{4 V_{\pi}^{2}} G Z_{P D} Z_{\text {in }} P_{o} P_{R F} \cos \Omega \tau
$$

Where $Z_{P D}$ and $Z_{\text {in }}$ are the output impedance of the photo-detector and input impedance of the MZMs, respectively, $P_{o}$ and $P_{R F}$ are the optical and RF input power respectively, $V_{\pi}$ is the MZMs' half wave voltage, $\tau$ is the delay caused by the SMF fiber, and $\Omega$ is the RF frequency. The factor $G$ is defined as $G=r G_{P D} L_{1} L_{2}$ where $r$ and $G_{P D}$ are the photo-detector responsivity and gain respectively and $L_{1}$ and $L_{2}$ are the MZM1 and MZM2 insertion loss respectively [8].

Fig. 2a presents the measured results along with the predicted response. Excellent agreement is evident. The DC level measured at the output can clearly be seen to vary sinusoidaly as the input frequency is varied. Fig. $2 b$ presents the measured frequency obtained by taking the inverse of Eq. (3) of the measured result. The implied frequency clearly follows the correct trend.

\section{Sensitivity Improvement}

In Section II, we described the operation of the photonic IFM receiver. To reduce the noise and increase the sensitivity of this system, one possible solution is to use lock-in amplifier. Fig. 3 shows the proposed block diagram of the receiver. Two optical carriers $\left(\lambda_{1}\right.$ and $\left.\lambda_{2}\right)$ are provided by a laser array and these wavelengths are switched using the first modulator (MZM1). The MZM1 is biased at $V_{\max }$ and $V_{\text {null }}$ with DC offset of $V_{q}$. A portion of this voltage is also used to feed the lock-in amplifier. The output of the MZM1 is then mixed with the RF signal using the MZM2 (biased at $V_{q}$ ). The modulated signal is delayed using a cascaded grating which provided different delay for $\lambda_{1}$ and $\lambda_{2}$. The delayed signal is again modulated, low-pass filtered and detected. The output of the photo-detector connected to the lock-in amplifier. Using two different wavelengths, different delays resulting in different output voltages 
can be achieved It is this difference in output voltage which is measured by the lock-in amplifier.

\section{Results and Discussion}

Fig.4a shows the measured lock-in output voltage as a function of frequency using the setup in Fig 3. In this instance, the input RF power is reduced to only $0 \mathrm{dBm}$ (compared to $16 \mathrm{dBm}$ in Figure 2a). The results of Fig.4a clearly shows the expected sinusoidal behavior predicted in Section II, Due to the absence of a DC offset, it is clear that the system remains sensitive even with this significant reduction of RF input power. Fig. $4 \mathrm{~b}$ presents the interpreted frequency as a function of RF frequency. To increase the operation frequency, the optical fiber length could be reduced through integration.

\section{Conclusion}

A simple technique to improve the sensitivity of a photonic instantaneous frequency measurement receiver using low-cost, low-frequency photo-detectors is proposed and practically demonstrated. The introduction of the lock-in amplification allows RF input power can be reduced by $16 \mathrm{~dB}$ when compared to the un-locked system while still maintaining sensitivity. Formal quantification of the noise floor of the system and extension to $\mathrm{GHz}$ frequencies is currently under investigation.

\section{References}

[1] H. Gruchala, and M. Czyzewski, "The instantaneous frequency measurement receiver in the complex electromagnetic environment," in Proceedings of International Conference on Microwave, RADAR, and Wireless. Communications (MIKON2004), vol. 1, pp. 155-158, May 2004.

[2] J. B. Y. Tsui, "Instantaneous frequency measurement receiver with digital processing," United States Patent 4633516, 30 Dec. 1986.

[3] R. B. Sanderson, and J. B. Y. Tsui, "Instantaneous frequency measurement receiver with bandwidth improvement through phase shifted sampling of real signals," United States Patent 5109188, 28 Apr. 1992.

[4] M. Aikawa, and H. Oqawa, "Double-sided MICs and their applications," IEEE Microwave Theory Tech., vol. 37, no. 2, pp. 406-413, Feb. 1989..

[5] L. V. T. Nguyen, and D. B. Hunter, "A photonic technique for microwave frequency measurement," IEEE Photon. Tech. Lett., vol. 18, no. 10, pp. 1188-1190, May 2006.

[6] J. Capmany, B. Ortega, D. Pastor, "A tutorial on microwave photonic filters," J. Lightwave Tech., vol. 24, no. 1, pp. 201-229, Jan. 2006.

[7] R. A. Minasian, "Photonic signal processing of microwave signals," IEEE Trans. Microwave Theory Tech., vol. 54, no. 2, pp. 832-846, Feb. 2006.

[8] N.Sarkhosh, H.Emami, L.Bui and A. Mitchell "Reduce cost Photonic Instantaneous Frequency Measurement System," IEEE Photon. Tech. Lett, Vol 20, NO.18, Sep 2008 
Figures

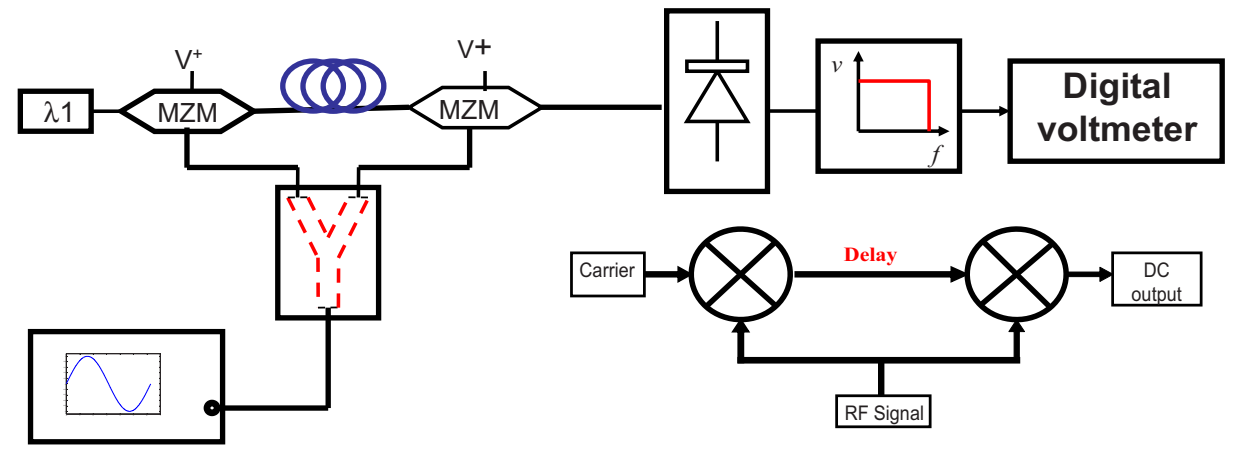

Fig. 1 Block diagram and Experimental set up of the photonic IFM system
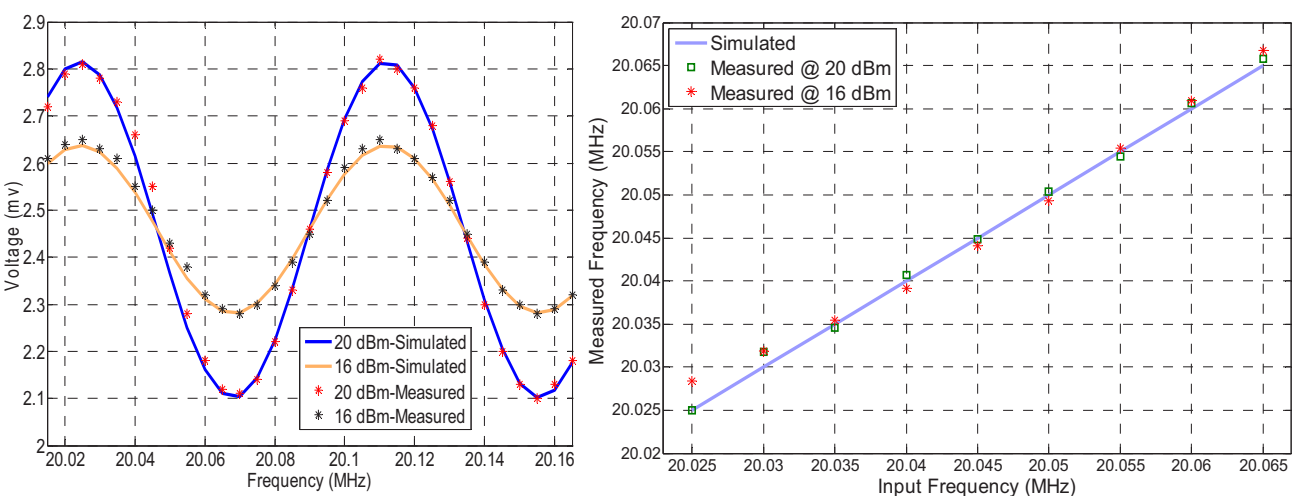

Fig. 2 a) Measured and predicted IFM output voltage, b) Measured and predicted frequency

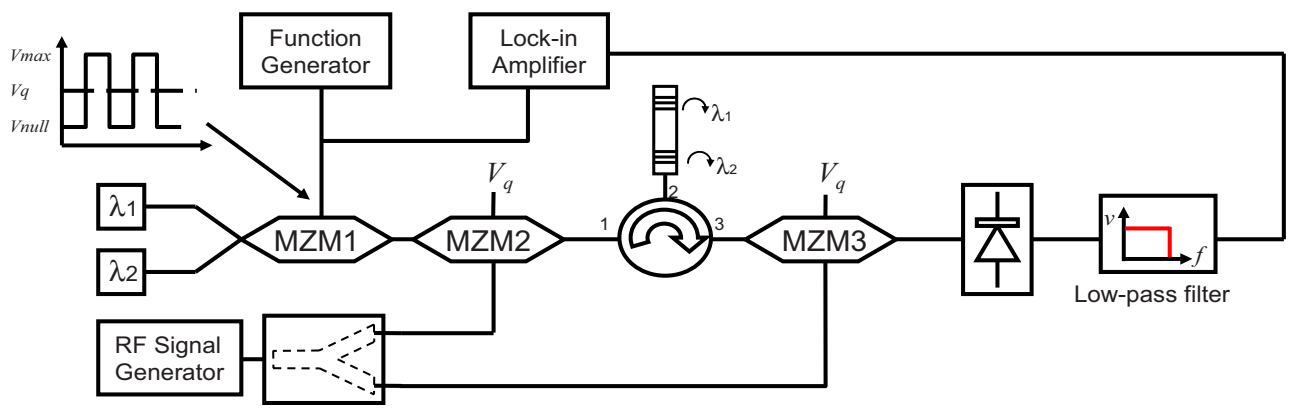

Fig. 3 Experimental set up of the photonic IFM with Lock-in Amplifier
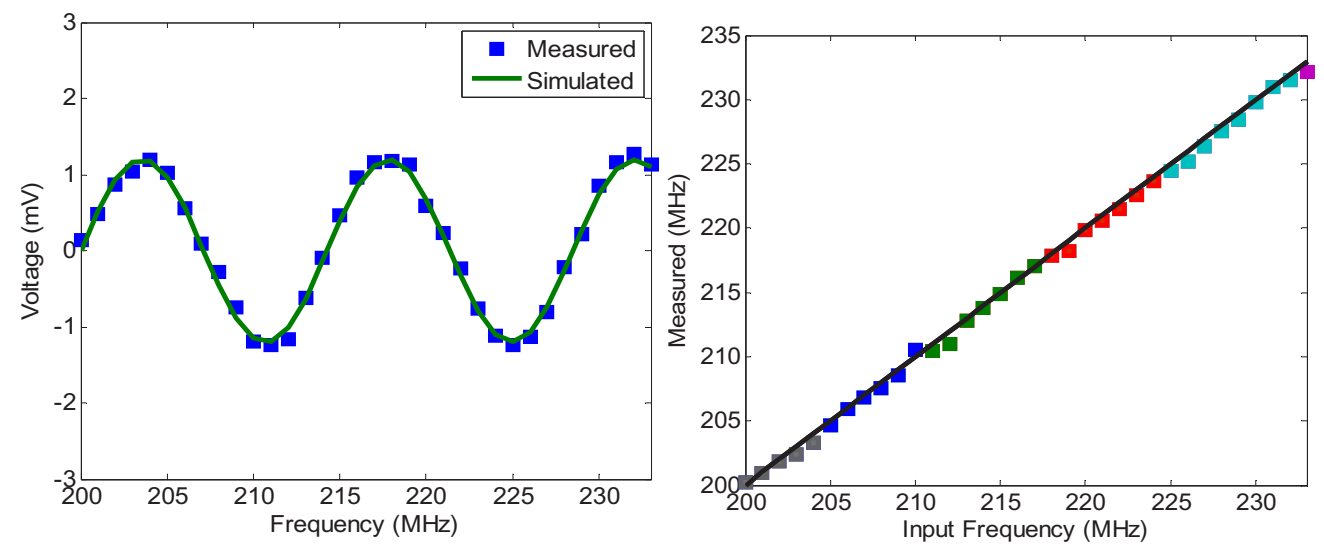

Fig. 4 a) Measured (lock-in output) and predicted IFM output voltage, b) Measured and predicted frequency 